\title{
Synthesis of 2-Quinuclidonium by Eliminating Water: Experimental Quantification of the High Basicity of Extremely Twisted Amides
}

Tony Ly, Michael Krout, Don K. Pham, Kousuke Tani, Brian M. Stoltz, and Ryan R. Julian

Contribution from the Department of Chemistry, University of California, Riverside, California, 92521, and the Division of Chemistry and Chemical Engineering, California Institute of Technology, Pasadena, California, 91125 (USA)

\section{Table of Contents:}

$\begin{array}{ll}\text { Materials and Methods } & \text { S2 }\end{array}$

Preparative Procedures $\quad$ S3

Computational Results $\quad$ S15

$\begin{array}{ll}\text { Mass Spectra of Isotopically Labeled Compounds } & \text { S18 }\end{array}$

$\begin{array}{ll}\text { Extended Kinetic Method Plots } & \text { S20 }\end{array}$ 


\section{Materials and Methods.}

\section{Organic Synthesis}

Unless stated otherwise, reactions were conducted in flame-dried glassware under an atmosphere of nitrogen using anhydrous solvents passed through activated alumina columns under argon. All commercially obtained reagents were used as received. Hexamethylphosphoramide was distilled from $\mathrm{CaH}_{2}$ and stored in a schlenk tube under argon. 3- $d$-cyclopentenone ${ }^{\mathrm{i}}$ and 6,6,7,7tetramethyl-2-quinuclidone ${ }^{\mathrm{ii}}$ were prepared by known methods. Sodium azide $\left(1{ }^{15} \mathrm{~N}, 98\right.$ atom\% $\left.{ }^{15} \mathrm{~N}\right)$ and acetic acid $\left(1,2-{ }^{13} \mathrm{C}_{2}, 99\right.$ atom\% $\left.{ }^{13} \mathrm{C}\right)$ were purchased from Cambridge Isotope Laboratories. Lithium aluminum deuteride (98 atom\% $d$ ) was purchased from Aldrich. Reaction temperatures were controlled using an IKAmag temperature modulator, and unless stated otherwise, reactions were performed at $23{ }^{\circ} \mathrm{C}$. Thin-layer chromatography (TLC) was conducted with E. Merck silica gel 60 F254 pre-coated plates, $(0.25 \mathrm{~mm})$ and visualized using a combination of UV, anisaldehyde, ceric ammonium molybdate, and potassium permanganate staining. ICN silica gel (particle size 0.032-0.063 mm) was used for flash column chromatography. ${ }^{1} \mathrm{H}$ NMR spectra were recorded on a Varian Mercury 300 (at $300 \mathrm{MHz}$ ), or a Varian Inova 500 (at $500 \mathrm{MHz}$ ), and are reported relative to $\mathrm{Me}_{4} \mathrm{Si}(\delta 0.0)$. Data for ${ }^{1} \mathrm{H}$ NMR spectra are reported as follows: chemical shift $(\delta \mathrm{ppm})$, multiplicity, coupling constant $(\mathrm{Hz})$ and integration. ${ }^{13} \mathrm{C}$ NMR spectra were recorded on a Varian Mercury 300 (at $75 \mathrm{MHz}$ ), or a Varian Inova 500 (at $125 \mathrm{MHz}$ ), and are reported relative to $\mathrm{Me}_{4} \mathrm{Si}(\delta 0.0)$. Data for ${ }^{13} \mathrm{C}$ NMR spectra are reported in terms of chemical shift, multiplicity, and coupling constant. ${ }^{2} \mathrm{H}$ NMR spectra were recorded on a Varian Inova 500 (at $76 \mathrm{MHz}$ ) and are reported relative to $\mathrm{Me}_{4} \mathrm{Si}(\delta 0.0)$. Data for ${ }^{2} \mathrm{H}$ NMR spectra are reported in terms of chemical shift and multiplicity. IR spectra were recorded on a Perkin Elmer Spectrum BXII spectrometer and are reported in terms of frequency of absorption $\left(\mathrm{cm}^{-1}\right)$. High resolution mass spectra were obtained from the California Institute of Technology Mass Spectral Facility.

\section{Extended Kinetic Method and Gas Phase Synthesis}

All mass spectra were obtained using an LTQ linear ion trap mass spectrometer (Thermo Electron, Waltham, MA) equipped with a standard electrospray ionization source. Voltages were optimized to maximize the $\left[\mathbf{1}+\mathrm{H}^{+}+\mathrm{B}_{\mathrm{ref}}\right]$ dimer peak intensities for kinetic method experiments. All reference bases were purchased from Sigma-Aldrich (St. Louis, MO) and were used without further purification. 
To minimize hydrolysis of $\mathbf{1} \cdot \mathbf{H B F}_{\mathbf{4}}$, samples containing $300 \mu \mathrm{M}$ of $\mathbf{1}$ and reference base were prepared with dry acetonitrile unless otherwise noted and immediately infused into the electrospray source. The noncovalently bound dimers were then isolated and subjected to CID at normalized collision energies (relative to instrument calibration and $\mathrm{m} / \mathrm{z}$ ) ranging from $18 \%$ to $85 \%$. These percentages correspond to excitation voltage amplitudes of $0.00641 \mathrm{~V}$ to $0.0303 \mathrm{~V}$ for a $100 \mathrm{~m} / \mathrm{z}$ ion. To obtain tandem MS data, $30 \mu \mathrm{M}$ solutions were prepared and analyzed as above under standard instrument tune conditions. Amino acid deriviatives were prepared by either allowing a sample sufficient time to hydrolyze (ca. 30 minutes) or by addition of deionized water in molar excess.

Proton affinities were calculated using hybrid density functional theory as implemented in Gaussian 03 Version 6.1 Revision D.01. Candidate structures were built using GaussView 3.0 and then submitted for optimization and vibrational frequency calculation at the B3LYP/6-311++G** level. Total energies, zero point energies (ZPE), and thermal corrections were obtained from the optimization/frequency output. Zero point corrections were scaled by an empirical factor of 0.9877

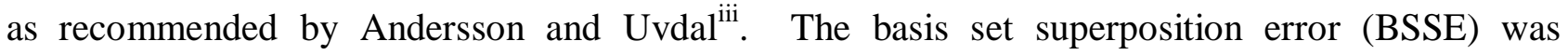
calculated using the counterpoise (CP) method of Boys and Bernardi ${ }^{\text {iv }}$.

\section{Preparative Procedures.}

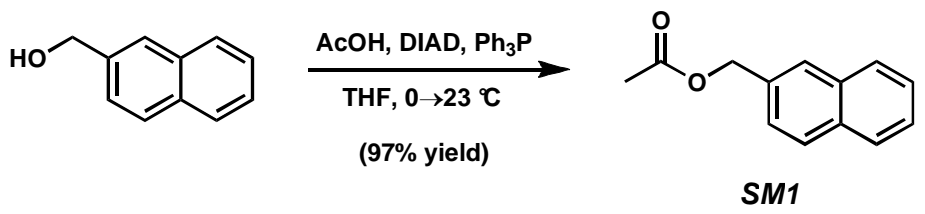

Naphthalen-2-ylmethyl acetate (SM1); CAS \# 35480-23-0. $\mathrm{Ph}_{3} \mathrm{P}(0.787$ g, 3.0 mmol, 1.5 equiv) and 2-napthalenylmethanol ( $0.475 \mathrm{~g}, 3.0 \mathrm{mmol}, 1.5$ equiv) were dissolved in THF (13.4 mL, $0.15 \mathrm{M})$. Acetic acid $\left(114 \mu \mathrm{L}, 2.0 \mathrm{mmol}, 1.0\right.$ equiv) was added and the solution was cooled to $0{ }^{\circ} \mathrm{C}$. DIAD (591 $\mu \mathrm{L}, 3.0 \mathrm{mmol}, 1.5$ equiv) dissolved in THF ( $1 \mathrm{~mL}$ ) was added dropwise over 5 min via positive pressure cannulation. After $1 \mathrm{~h}$ the reaction was quenched with $5 \mathrm{~mL}$ sat'd $\mathrm{NaHCO}_{3}$, extracted with hexanes $(3 \times 20 \mathrm{~mL})$, the organics were dried over $\mathrm{MgSO}_{4}$, filtered, and concentrated under reduced pressure to an off-white solid. The crude material was purified by flash chromatography $\left(\mathrm{SiO}_{2}, 15: 1 \rightarrow\right.$ 9:1 $\mathrm{Hex}-\mathrm{Et}_{2} \mathrm{O}, \mathrm{PhMe}$ load) to afford naphthalen-2-ylmethyl acetate

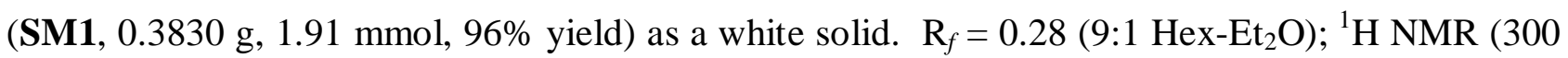


$\mathrm{MHz}, \mathrm{CDCl}_{3}$ ): $\delta$ 7.89-7.85 (comp. m, 4H), 7.53-7.47 (comp. m, 3H), 5.30 (s, 2H), 2.16 (s, $3 \mathrm{H}$ ); ${ }^{13} \mathrm{C}$ NMR (125 MHz, $\left.\mathrm{CDCl}_{3}\right): \delta$ 171.1, 133.5, 133.4, 133.3, 128.5, 128.1 (2C), 127.9, 127.5 (2C), 126.5, 126.4, 126.1, 66.6, 21.2; IR (neat film, NaCl): 3055, 2953, 1736, 1378, 1364, 1248, 1030, 951, 896, 863, 822, 744, $480 \mathrm{~cm}^{-1}$; HRMS-EI $(\mathrm{m} / \mathrm{z})$ : $[\mathrm{M}]^{+\bullet}$ calc'd for $\left[\mathrm{C}_{13} \mathrm{H}_{12} \mathrm{O}_{2}\right]^{+\bullet}, 200.0837$; found, 200.0844; $\mathrm{mp}=53-55{ }^{\circ} \mathrm{C}$.

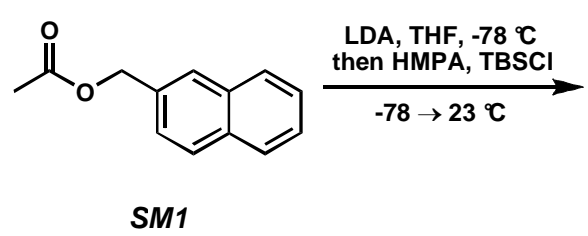

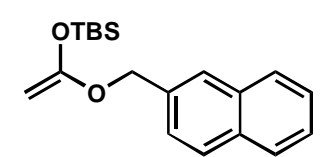

SM2

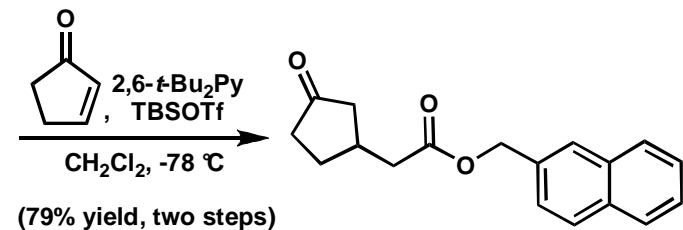

SM3

Naphthalen-2-ylmethyl 2-(3-oxocyclopentyl)ethanoate (SM3). To a cooled solution of $i$ $\mathrm{Pr}_{2} \mathrm{NH}\left(341 \mu \mathrm{L}, 2.44 \mathrm{mmol}, 1.15\right.$ equiv) in THF $(2.12 \mathrm{~mL}, 1 \mathrm{M})$ at $0{ }^{\circ} \mathrm{C}$ was added $n$-BuLi ( $2.5 \mathrm{M}$ in hexane) dropwise. After stirring for $15 \mathrm{~min}$, the solution was cooled to $-78{ }^{\circ} \mathrm{C}$ and a solution of 2-naphthylmethyl acetate (SM1, $424.0 \mathrm{mg}, 2.12 \mathrm{mmol}, 1.0$ equiv) in THF (1 mL) was added dropwise via positive pressure cannulation. After $15 \mathrm{~min}$, HMPA (332 $\mu \mathrm{L}, 1.91 \mathrm{mmol}, 0.9$ equiv), then TBSCl (351.0 mg, $2.33 \mathrm{mmol}, 1.1$ equiv) in THF $(0.80 \mathrm{~mL})$ were added and the cooling bath was removed. The reaction was warmed to ambient temperature and concentrated under reduced pressure. The resulting thick oil was dissolved in 9:1 $\mathrm{Hex}_{-} \mathrm{Et}_{2} \mathrm{O}(50 \mathrm{~mL})$ and washed with distilled water ( 3 x $20 \mathrm{~mL}, \mathrm{pH} \sim 7$ ) and sat'd brine. The organic layer was dried over $\mathrm{MgSO}_{4}$, filtered, and concentrated under reduced pressure. The resulting yellow oil solidified after several hours under high vacuum to afford TBS-silylenol ether SM2 $(650.9 \mathrm{mg})$ which was used without further purification in the subsequent reaction. $\mathrm{R}_{f}=$ unstable to $\mathrm{SiO}_{2}$.

To a solution of SM2 (1.2 equiv), cyclopentenone (145 $\mu \mathrm{L}, 1.72 \mathrm{mmol}, 1.0$ equiv), and 2,6ditertbutylpyridine ( $465 \mu \mathrm{L}, 2.07 \mathrm{mmol}, 1.2$ equiv) in $\mathrm{CH}_{2} \mathrm{Cl}_{2}(20.7 \mathrm{~mL}, 0.1 \mathrm{M})$ cooled to $-78{ }^{\circ} \mathrm{C}$ was added a solution of TBSOTf ( $475 \mu \mathrm{L}, 2.07 \mathrm{mmol}, 1.2$ equiv) in $\mathrm{CH}_{2} \mathrm{Cl}_{2}(2.1 \mathrm{~mL})$ dropwise over 15 min. Following consumption of cyclopentenone by TLC (15 min), the cooling bath was removed and the reaction was quenched with $15 \mathrm{~mL}$ of $3 \%$ aq $\mathrm{HCl}$. After stirring for 30 min the layers were separated and the aqueous layer was extracted with $\mathrm{CH}_{2} \mathrm{Cl}_{2}(3 \times 25 \mathrm{~mL})$, the organics dried over $\mathrm{MgSO}_{4}$, filtered, and concentrated under reduced pressure to a yellow solid. The crude product was purified by flash chromatography $\left(\mathrm{SiO}_{2}, 9: 1 \rightarrow 4: 1 \rightarrow 3: 1 \mathrm{Hex}\right.$-EtOAc, dry load) to afford naphthalen-2-ylmethyl 2-(3-oxocyclopentyl)ethanoate (SM3, $385.4 \mathrm{mg}, 1.37 \mathrm{mmol}$ ) as a light 
yellow oil. $\mathrm{R}_{f}=0.23\left(1: 1 \mathrm{Hex}_{-} \mathrm{Et}_{2} \mathrm{O}\right) ;{ }^{1} \mathrm{H} \mathrm{NMR}\left(300 \mathrm{MHz}, \mathrm{CDCl}_{3}\right)$ : $\delta$ 7.87-7.82 (comp. m, 4H), 7.53-7.49 (comp. m, 2H), 7.45 (dd, $J=8.5,1.9 \mathrm{~Hz}, 1 \mathrm{H}), 5.30(\mathrm{~s}, 2 \mathrm{H}), 2.70-2.57$ (m, 1H), 2.55 (d, $J$ $=1.1 \mathrm{~Hz}, 1 \mathrm{H}), 2.53(\mathrm{~d}, J=2.9 \mathrm{~Hz}, 1 \mathrm{H}), 2.51-2.45$ (m, 1H), 2.38-2.11 (comp. m, 3H), 1.90 (ddd, $J=$ 18.1, 9.8, 1.1 Hz, 1H), 1.66-1.51 (m, 1H); $\left.{ }^{13} \mathrm{C} \mathrm{NMR} \mathrm{(75} \mathrm{MHz,} \mathrm{CDCl}_{3}\right): \delta 218.4,172.0,133.3(2 \mathrm{C})$, 133.2, 128.6, 128.1, 127.9, 127.7, 126.5 (2C), 126.0, 66.7, 44.7, 39.9, 38.4, 33.6, 29.4; IR (neat film, $\mathrm{NaCl}): 3049,2956,1737,1271,116,817 \mathrm{~cm}^{-1}$; HRMS-EI $(\mathrm{m} / z):[\mathrm{M}]^{+\bullet}$ calc'd for $\left[\mathrm{C}_{18} \mathrm{H}_{18} \mathrm{O}_{3}\right]^{+\bullet}$, 282.1256; found, 282.1257.
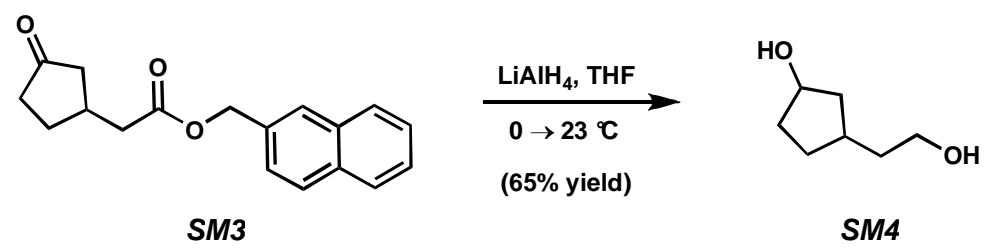

3-(2-hydroxyethyl)cyclopentanol (SM4). To a slurry of $\mathrm{LiAlH}_{4}(74.3 \mathrm{mg}, 1.96$ mmol, 4.0 equiv) in THF (4.9 mL, 0.1M) at $0{ }^{\circ} \mathrm{C}$ was added ketoester SM3 (138.1 mg, $0.498 \mathrm{mmol}, 1.0$ equiv) in $1.0 \mathrm{~mL}$ THF. The cooling bath was removed and the reaction was stirred for $2.5 \mathrm{~h}$ at ambient temperature. The reaction was then cooled to $0{ }^{\circ} \mathrm{C}$ and carefully quenched by slow addition of $\mathrm{Na}_{2} \mathrm{SO}_{4} \bullet 10 \mathrm{H}_{2} \mathrm{O}$. When gas evolution had ceased, the flask was diluted up to $25 \mathrm{~mL}$ with EtOAc and stirred vigorously at ambient temperature for $2 \mathrm{~h}$. The fine precipitate was then filtered throught celite, washing with excess EtOAc, and the resulting filtrate was concentrated under reduced pressure to an off-white solid. This residue was purified by flash chromatography $\left(\mathrm{SiO}_{2}\right.$, 2:1 $\rightarrow$ 1:0 EtOAc-Hex) to afford 1:1 mixture of diastereomers of 3-(2-hydroxyethyl)cyclopentanol (SM4, $42.6 \mathrm{mg}, 0.320 \mathrm{mmol}, 65 \%$ yield) as a colorless oil. $\mathrm{R}_{f}=0.15$ (3:1 EtOAc-Hex); ${ }^{1} \mathrm{H}$ NMR $\left(500 \mathrm{MHz}, \mathrm{CD}_{3} \mathrm{OD}\right.$ ): $\delta 4.26$ (anti diastereomer, ddd, $\left.J=5.6,5.6,2.9,2.9 \mathrm{~Hz}, 0.47 \mathrm{H}\right), 4.21$ (syn diastereomer, dddd, $J=5.9,5.9,5.9,5.9 \mathrm{~Hz}, 0.53 \mathrm{H}), 3.56(\operatorname{app} \mathrm{t}, J=6.8 \mathrm{~Hz}, 2 \mathrm{H}), 2.20(\mathrm{ddd}, J=$ $16.4,7.8,7.8 \mathrm{~Hz}, 0.53 \mathrm{H}), 2.14$ (ddd, $J=14.2,7.6,7.6 \mathrm{~Hz}, 0.53 \mathrm{H}), 1.98-1.90$ (comp. m, 1.5H), 1.811.72 (comp. m, 1.5H), 1.65-1.60 (comp. m, 1.5H), 1.59-1.51 (comp. m, 1.5H), 1.44-1.31 (m, 1H), 1.19-1.12 (m, 1H); ${ }^{13} \mathrm{C}$ NMR (125 MHz, CD $\left.3 \mathrm{OD}\right): \delta 74.1$ (syn), 62.2, 62.1 (syn), 43.1, 42.9 (syn), 40.6 (syn), 40.1, 36.1 (syn), 35.8 (syn), 35.6, 35.2, 31.5, 31.2 (syn); IR (neat film, NaCl): 3323 (broad), 2931, 2864, 1434, 1344, 1052, $1013 \mathrm{~cm}^{-1}$; HRMS-EI $(\mathrm{m} / z)$ : $[\mathrm{M}]^{+\bullet}$ calc'd for $\left[\mathrm{C}_{7} \mathrm{H}_{14} \mathrm{O}_{2}\right]^{+\bullet}$, 130.0994; found 130.0994. 


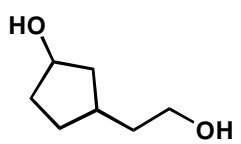

SM4

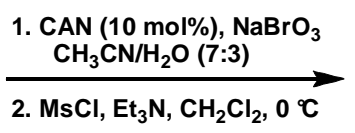

(83\% yield, 2 steps)

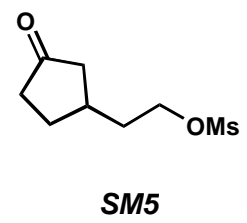

2-(3-oxocyclopentyl)ethyl methanesulfonate (SM5). ${ }^{\mathrm{v}} \mathrm{CH}_{3} \mathrm{CN}(2.8 \mathrm{~mL}, 0.167 \mathrm{M})$, CAN (36.9 mg, $0.673 \mathrm{mmol}, 0.1$ equiv), $\mathrm{NaBrO}_{3}$ (101.5 mg, 0.673 mmol, 1.0 equiv), and distilled $\mathrm{H}_{2} \mathrm{O}$ $(1.2 \mathrm{~mL})$ were added to diol $\mathbf{S M 4}(87.6 \mathrm{mg}, 0.673 \mathrm{mmol}, 1.0$ equiv) in a vial and stirred vigorously. Following consumption of diol SM4 by TLC (ca. $6 \mathrm{~h}$ ), the reaction was concentrated under reduced pressure. The resulting slurry was taken up in $10 \mathrm{~mL} \mathrm{H}_{2} \mathrm{O}$, extracted with EtOAc ( 3 x $25 \mathrm{~mL}$ ), dried over $\mathrm{Na}_{2} \mathrm{SO}_{4}$, filtered, and concentrated under reduced pressure to afford a crude yellow oil (85.5 mg).

The resulting crude material was dissolved in $\mathrm{CH}_{2} \mathrm{Cl}_{2}(1.35 \mathrm{~mL}, 0.5 \mathrm{M})$, cooled to $0{ }^{\circ} \mathrm{C}$, and $\mathrm{MsCl}\left(77.4 \mu \mathrm{L}, 1.0 \mathrm{mmol}, 1.5\right.$ equiv) and $\mathrm{Et}_{3} \mathrm{~N}$ (167 $\mu \mathrm{L}, 1.2 \mathrm{mmol}, 1.8$ equiv) were added sequentially. After $5 \mathrm{~min}$, the reaction was quenched with sat'd aq. $\mathrm{NaHCO}_{3}(1 \mathrm{~mL})$ and diluted up to $10 \mathrm{~mL}$ with $\mathrm{CH}_{2} \mathrm{Cl}_{2}$. The biphasic solution was further diluted with sat'd $\mathrm{NaHCO}_{3}(2 \mathrm{~mL})$ and sat'd brine $(2 \mathrm{~mL})$, the layers were separated, and the aqueous was extracted with $\mathrm{CH}_{2} \mathrm{Cl}_{2}(3 \times 10$ $\mathrm{mL}$ ). The combined organics were dried over $\mathrm{MgSO}_{4}$, filtered, and concentrated to a light yellow solid under reduced pressure. This residue was purified by flash chromatography $\left(\mathrm{SiO}_{2}, 2: 1 \rightarrow 1: 2\right.$ Hex-EtOAc, dry load) to afford 2-(3-oxocyclopentyl)ethyl methanesulfonate (SM5, $115.4 \mathrm{mg}$, $0.560 \mathrm{mmol}, 83 \%$ yield over two steps) as a colorless oil. $\mathrm{R}_{f}=0.31$ (3:1 EtOAc-Hex); ${ }^{1} \mathrm{H}$ NMR (300 MHz, $\mathrm{CDCl}_{3}$ ): $\delta 4.29$ (app dt, $J=6.4,2.4,2.4 \mathrm{~Hz}, 2 \mathrm{H}$ ), 3.02 (s, 3H), 2.51-2.42 (m, 1H), 2.402.12 (comp. m, 4H), 1.95-1.89 (comp. m, 2H), 1.84 (ddd, $J=17.6,7.7,1.3 \mathrm{~Hz}, 1 \mathrm{H}$ ), 1.64-1.48 (m, $1 \mathrm{H}) ;{ }^{13} \mathrm{C}$ NMR $\left(75 \mathrm{MHz}, \mathrm{CDCl}_{3}\right): \delta 218.3,68.2,44.8,38.5,37.6,35.0,33.8,29.4$; IR (neat film, $\mathrm{NaCl}): 3023,2935,1737,1350,1173,954 \mathrm{~cm}^{-1}$; HRMS-EI $(\mathrm{m} / \mathrm{z})$ : $[\mathrm{M}]^{+\bullet}$ calc'd for $\left[\mathrm{C}_{8} \mathrm{H}_{14} \mathrm{O}_{4} \mathrm{~S}\right]^{+*}$, 206.0613; found 206.0622.

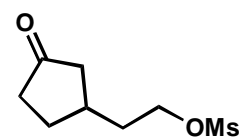

SM5

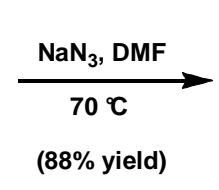

(88\% yield)

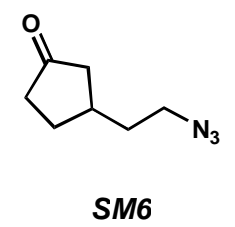

3-(2-azidoethyl)cyclopentanone (SM6). To a solution of mesylate SM5 (50.2 mg, 0.243 mmol, 1.0 equiv) in DMF ( $0.50 \mathrm{~mL}, 0.5 \mathrm{M})$ was added $\mathrm{NaN}_{3}(17.4 \mathrm{mg}, 0.268 \mathrm{mmol}, 1.1$ equiv), and 
the mixture was warmed to $70{ }^{\circ} \mathrm{C}$ until consumption of SM5 by TLC. The reaction was cooled to 0 ${ }^{\circ} \mathrm{C}$ and stirred for $15 \mathrm{~min}$, followed by dilution with $\mathrm{Et}_{2} \mathrm{O}$. The suspension was filtered through a plug of celite with $\mathrm{Et}_{2} \mathrm{O}$, concentrated under reduced pressure, and purified by flash chromatography $\left(\mathrm{SiO}_{2}, 6: 1 \rightarrow\right.$ 3:1 $\mathrm{Hex}-\mathrm{Et}_{2} \mathrm{O}, \mathrm{PhMe}$ load) to afford 3-(2-azidoethyl)cyclopentanone (SM6, $32.8 \mathrm{mg}, 0.214 \mathrm{mmol}, 88 \%$ yield) as a colorless oil. $\mathrm{R}_{f}=0.25$ (3:1 Hex-EtOAc); characterization data for this compound have previously been reported. ${ }^{\mathrm{vi}}$

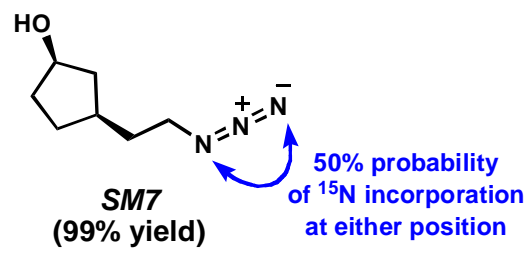

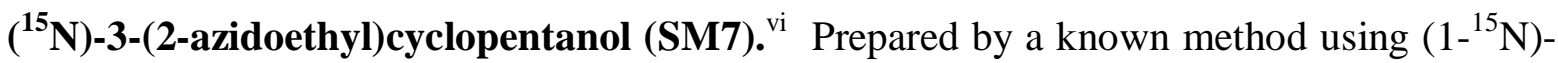
$\mathrm{NaN}_{3}$. The reaction was purified by flash chromatography $\left(\mathrm{SiO}_{2}, 3: 1 \rightarrow 1: 1 \mathrm{Hex}_{-} \mathrm{Et}_{2} \mathrm{O}, \mathrm{PhMe}\right.$ load $)$ to afford ${ }^{15} \mathrm{~N}$-labeled azidoalcohol SM7 (186.1 mg, $1.19 \mathrm{mmol}, 99 \%$ yield) as colorless oil. $\mathrm{R}_{f}=$ 0.14 (1:1 Hex-Et $2 \mathrm{O}$ ); IR (neat film, NaCl): 3344, 2946, 2868, 2074, 1339, $1243 \mathrm{~cm}^{-1}$; HRMS-FAB $(m / z):[\mathrm{M}+\mathrm{H}]^{+}$calc'd for $\left[\mathrm{C}_{7} \mathrm{H}_{14} \mathrm{~N}_{2} \mathrm{O}^{15} \mathrm{~N}\right]^{+}, 157.1107$; found 157.1141. All other characterization data are identical to that reported.

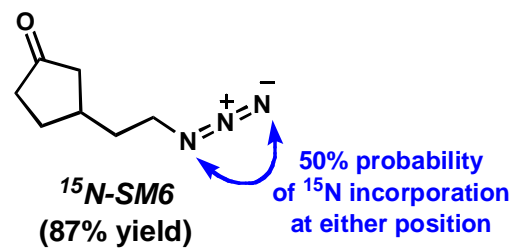

$\left({ }^{15} \mathrm{~N}\right)-3-\left(2\right.$-azidoethyl)cyclopentanone $\left({ }^{15} \mathrm{~N}-\mathrm{SM6}\right) .{ }^{\mathrm{vi}}$ Prepared by a known method. The reaction was purified by flash chromatography $\left(\mathrm{SiO}_{2}, 6: 1 \rightarrow 3: 1 \mathrm{Hex}_{-}-\mathrm{Et}_{2} \mathrm{O}\right)$ to afford ketoazide ${ }^{\mathbf{1 5}} \mathbf{N}-$ SM6 (155.5 mg, $1.00 \mathrm{mmol}, 87 \%$ yield) as colorless oil. $\mathrm{R}_{f}=0.26$ (1:1 Hex-Et $2 \mathrm{O}$ ); IR (neat film, $\mathrm{NaCl}): 2931,2873,2076,1740,1242,1160 \mathrm{~cm}^{-1}$. All other characterization data are identical to that reported. 


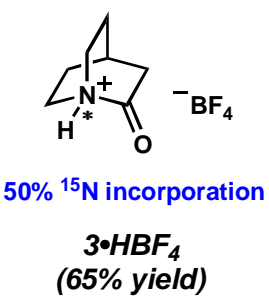

1- ${ }^{15} \mathbf{N}$-2-quinuclidonium tetrafluoroborate $\left(3 \cdot \mathbf{H B F}_{4}\right){ }^{\mathrm{vi}}$ Prepared by a known method. The crude reaction precipitate was transferred to a glovebox and recrystallized $2 \mathrm{X}$ by slow diffusion of $\mathrm{Et}_{2} \mathrm{O}$ into $\mathrm{CH}_{3} \mathrm{CN}$ at $-20{ }^{\circ} \mathrm{C}$ to afford $\mathbf{3} \cdot \mathbf{H B F}_{4}(127.7 \mathrm{mg}, 0.598 \mathrm{mmol}, 65 \%$ yield) as white needles. HRMS-FAB $(m / z):[\mathrm{M}+\mathrm{H}]^{+}$calc'd for $\left[\mathrm{C}_{7} \mathrm{H}_{12} \mathrm{O}^{15} \mathrm{~N}\right]^{+}, 127.0889$; observed 127.0855; $[\mathrm{M}+\mathrm{H}]^{+}$calc'd for $\left[\mathrm{C}_{7} \mathrm{H}_{12} \mathrm{NO}\right]^{+}, 126.0919$; observed 126.0915; relative peak ratio = 1:1.

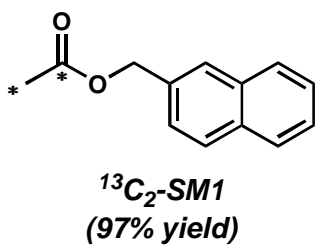

Naphthalen-2-ylmethyl $\left({ }^{13} \mathbf{C}_{2}\right)$-acetate $\left({ }^{13} \mathbf{C}_{2}\right.$-SM1): Prepared as above to afford ${ }^{13} \mathbf{C}_{2}$-SM1 (0.8326 g, $4.12 \mathrm{mmol}, 97 \%$ yield) as an off-white solid. ${ }^{1} \mathrm{H}$ NMR (300 $\left.\mathrm{MHz}, \mathrm{CDCl}_{3}\right): \delta 7.87-7.83$ (comp. m, 4H), 7.53-7.45 (comp. m, 3H), 5.27 (d, $\left.J_{H-13 C}=3.2 \mathrm{~Hz}, 2 \mathrm{H}\right), 2.13$ (dd, $J_{H-13 C}=129.7,6.9$ $\mathrm{Hz}) ;{ }^{13} \mathrm{C}$ NMR $\left(75 \mathrm{MHz}, \mathrm{CDCl}_{3}\right): \delta 170.9\left(\mathrm{~d}, J_{13 \mathrm{C}-13 \mathrm{C}}=59.4 \mathrm{~Hz}\right), 21.1\left(\mathrm{~d}, J_{13 \mathrm{C}-13 \mathrm{C}}=59.2 \mathrm{~Hz}\right)$; IR (neat film, $\mathrm{NaCl}$ ): 3054, 2955, 1693, 1360, 1276, 1218, 1024, 970, 951, 897, 864, 823, $744 \mathrm{~cm}^{-1}$; HRMS-EI $(\mathrm{m} / \mathrm{z})$ : $[\mathrm{M}]^{+\bullet}$ calc'd for $\left[\mathrm{C}_{11} \mathrm{H}_{12} \mathrm{O}_{2}{ }^{13} \mathrm{C}_{2}\right]^{+\bullet}, 202.0904$; found 202.0913; mp = 54-56 ${ }^{\circ} \mathrm{C}$.

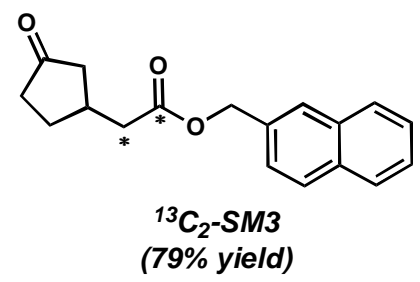

Naphthalen-2-ylmethyl 2-(3-oxocyclopentyl)- ${ }^{13} \mathrm{C}_{2}$-ethanoate $\left({ }^{13} \mathrm{C}_{2}\right.$-SM3). Prepared as above to afford ${ }^{13} \mathbf{C}_{2}$-SM3 (0.4204 g, 1.42 mmol, 79\% yield) as a light yellow oil. ${ }^{1} \mathrm{H}$ NMR (300 $\mathrm{MHz}_{\mathrm{CDCl}}$ ): $\delta$ 7.87-7.82 (comp. m, 4H), 7.53-7.49 (comp. m, 2H), 7.45 (dd, J=8.5, $\left.1.6 \mathrm{~Hz}, 1 \mathrm{H}\right)$, $5.30(\mathrm{~d}, J=3.2 \mathrm{~Hz}, 2 \mathrm{H}), 2.54\left(\mathrm{dddd}, J_{H-13 C}=129.2,6.9 \mathrm{~Hz}, J=10.9,2.0 \mathrm{~Hz}, 2 \mathrm{H}\right), 2.71-2.58(\mathrm{~m}$, 
1H), 2.49 (ddd, $\left.J=16.8,7.4 \mathrm{~Hz}, J_{H-13 \mathrm{C}}=1.3 \mathrm{~Hz}, 1 \mathrm{H}\right), 2.28-2.11$ (comp. m, 3H), 1.96 (dddd, $J=$ $\left.18.1,10.4,5.3 \mathrm{~Hz}, J_{H-13 C}=1.3 \mathrm{~Hz}, 1 \mathrm{H}\right), 1.67-1.50(\mathrm{~m}, 1 \mathrm{H}) ;{ }^{13} \mathrm{C} \mathrm{NMR}\left(75 \mathrm{MHz}, \mathrm{CDCl}_{3}\right): \delta 172.0(\mathrm{~d}$, $\left.J_{13 \mathrm{C}-13 \mathrm{C}}=57.2 \mathrm{~Hz}\right), 39.9\left(\mathrm{~d}, J_{13 \mathrm{C}-13 \mathrm{C}}=57.5 \mathrm{~Hz}\right.$ ); IR (neat film, $\left.\mathrm{NaCl}\right): 3054,2958,1740,1690,1403$, 1150, 1124, 818, $754 \mathrm{~cm}^{-1}$; HRMS-EI $(\mathrm{m} / \mathrm{z})$ : $[\mathrm{M}]^{+\bullet}$ calc'd for $\left[\mathrm{C}_{16} \mathrm{H}_{18} \mathrm{O}_{3}{ }^{13} \mathrm{C}_{2}\right]^{+\bullet}$, 284.1323; found 284.1322. All other characterization data are identical to above.

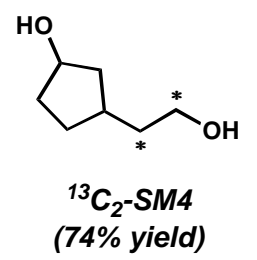

3-(2- ${ }^{13} \mathbf{C}_{2}$-hydroxyethyl)cyclopentanol $\left({ }^{13} \mathbf{C}_{2}\right.$-SM4). Prepared as above to afford $\sim 1: 1$ mixture of diastereomers of ${ }^{13} \mathbf{C}_{2}$-SM4 $\left(54.9 \mathrm{mg}, 0.415 \mathrm{mmol}, 74 \%\right.$ yield) as a colorless oil. ${ }^{1} \mathrm{H}$ NMR (300 MHz, $\mathrm{CD}_{3} \mathrm{OD}$ ): $\delta 4.26$ (dddd, $J=5.6,5.6,2.9,2.9 \mathrm{~Hz}, 0.44 \mathrm{H}$ ), 4.21 (dddd, $J=4.8,4.8$, $4.8,4.8,0.56 \mathrm{H}), 3.56\left(\mathrm{dddd}, J_{H-13 C}=140.2,6.9 \mathrm{~Hz}, J=6.9,2.4 \mathrm{~Hz}, 2 \mathrm{H}\right), 2.18-2.09(\mathrm{~m}, 1 \mathrm{H}), 2.0-1.7$ (comp. m, 3H), 1.65-1.49 (m, 1H), 1.46-1.28 (comp. m, 2H), 1.23-1.09 (m, 1H); ${ }^{13} \mathrm{C}$ NMR $(75$ $\left.\mathrm{MHz}, \mathrm{CD}_{3} \mathrm{OD}\right): \delta 62.2\left(\mathrm{~d}, J_{13 C-13 C}=37.3 \mathrm{~Hz}, 0.44 \mathrm{C}\right), 62.1\left(\mathrm{~d}, J_{13 C-13 \mathrm{C}}=37.3 \mathrm{~Hz}, 0.56 \mathrm{C}\right), 40.6(\mathrm{~d}$, $\left.J_{13 \mathrm{C}-13 \mathrm{C}}=37.3 \mathrm{~Hz}, 0.56 \mathrm{C}\right), 40.1\left(\mathrm{~d}, J_{13 \mathrm{C}-13 \mathrm{C}}=37.3 \mathrm{~Hz}, 0.44 \mathrm{C}\right)$. All other characterization data are identical to above.

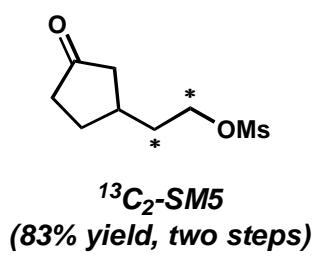

2-(3-oxocyclopentyl) $\left({ }^{13} \mathbf{C}_{2}\right)$-ethyl methanesulfonate $\left({ }^{13} \mathbf{C}_{2}\right.$-SM5). Prepared as above to afford ${ }^{13} \mathbf{C}_{2}$-SM5 (71.7 mg, $0.344 \mathrm{mmol}, 83 \%$ yield over two steps) as a colorless oil. ${ }^{1} \mathrm{H}$ NMR (300 $\mathrm{MHz}, \mathrm{CDCl}_{3}$ ): $\delta 4.30\left(\mathrm{dddd}, J_{H-13 C}=149.4,6.4 \mathrm{~Hz}, J=6.4,2.7 \mathrm{~Hz}, 2 \mathrm{H}\right), 3.03(\mathrm{~s}, 3 \mathrm{H}), 2.47$ (ddd, $J$ $\left.=17.8,7.5 \mathrm{~Hz}, J_{H-13 C}=1.0 \mathrm{~Hz}, 1 \mathrm{H}\right), 2.41-2.08$ (comp. m, $\left.5 \mathrm{H}\right), 1.85$ (dddd, $J=17.8,10.1,5.1 \mathrm{~Hz}$, $\left.J_{H-13 C}=1 \mathrm{~Hz}, 1 \mathrm{H}\right), 1.74-1.66(\mathrm{~m}, 1 \mathrm{H}), 1.63-1.52(\mathrm{~m}, 1 \mathrm{H}) ;{ }^{13} \mathrm{C} \mathrm{NMR}\left(75 \mathrm{MHz}, \mathrm{CDCl}_{3}\right): \delta 68.1(\mathrm{~d}$, $\left.J_{13 \mathrm{C}-13 \mathrm{C}}=37.9 \mathrm{~Hz}\right), 35.0\left(\mathrm{~d}, J_{13 \mathrm{C}-13 \mathrm{C}}=38.2 \mathrm{~Hz}\right)$; HRMS-EI $(\mathrm{m} / \mathrm{z}):[\mathrm{M}]^{+\bullet}$ calc'd for $\left[\mathrm{C}_{6} \mathrm{H}_{14} \mathrm{SO}_{4}{ }^{13} \mathrm{C}_{2}\right]^{+\bullet}$, 208.0680; found 208.0688. All other characterization data are identical to above. 


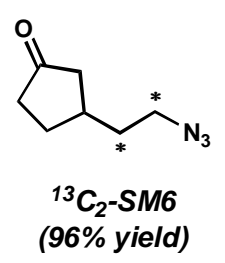

3-(2-azidoethyl)( $\left.{ }^{13} \mathbf{C}_{2}\right)$-cyclopentanone $\left({ }^{13} \mathbf{C}_{2}\right.$-SM6). Prepared as above to afford ${ }^{13} \mathbf{C}_{2}$ SM6 (28.5 mg, $0.184 \mathrm{mmol}, 96 \%$ yield) as a colorless oil. . ${ }^{1} \mathrm{H} \mathrm{NMR}\left(300 \mathrm{MHz}, \mathrm{CDCl}_{3}\right): \delta 3.36$ (app ddt, $\left.J_{H-13 C}=141.4,6.9 \mathrm{~Hz}, J=3.2 \mathrm{~Hz}, 2 \mathrm{H}\right), 2.44(\mathrm{dd}, J=17.8,8.0 \mathrm{~Hz}, 1 \mathrm{H}), 2.37-2.11$ (comp. m, 4H), 2.00-1.91 (m, 1H), 1.83 (ddd, $J=17.6,9.8,4.8 \mathrm{~Hz}, 1 \mathrm{H}), 1.62-1.49$ (comp. m, $2 \mathrm{H}) ;{ }^{13} \mathrm{C}$ NMR $\left(75 \mathrm{MHz}, \mathrm{CDCl}_{3}\right): \delta 50.0\left(\mathrm{~d}, J_{13 \mathrm{C}-13 \mathrm{C}}=36.8 \mathrm{~Hz}\right), 34.6\left(\mathrm{~d}, J_{13 \mathrm{C}-13 \mathrm{C}}=36.8 \mathrm{~Hz}\right)$. All other characterization data are identical to above.

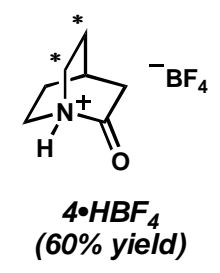

5,6- ${ }^{13} \mathbf{C}_{2}$-2-quinuclidonium tetrafluoroborate $\left(4 \cdot H B F_{4}\right)$. Prepared as above to afford $\mathbf{4} \cdot \mathrm{HBF}_{\mathbf{4}}$ (36.5 mg, $0.170 \mathrm{mmol}, 60 \%$ yield) as white needles. ${ }^{1} \mathrm{H}$ NMR (300 MHz, $\left.\mathrm{CD}_{3} \mathrm{CN}\right): \delta 7.99$ (br s, 1H), $3.69(\mathrm{~m}, 2 \mathrm{H}), 3.69\left(\mathrm{~m}, J_{H-13 C}=150.4 \mathrm{~Hz}, 2 \mathrm{H}\right), 2.97$ (app. d, $\left.J=5.4,3.3 \mathrm{~Hz}, 3 \mathrm{H}\right), 2.55-$ $2.47(\mathrm{~m}, 1 \mathrm{H}), 1.98(\mathrm{~m}, 2 \mathrm{H}), 1.98\left(\mathrm{~m}, J_{H-13 C}=135.4 \mathrm{~Hz}, 2 \mathrm{H}\right) ;{ }^{13} \mathrm{C} \mathrm{NMR}\left(75 \mathrm{MHz}, \mathrm{CD}_{3} \mathrm{CN}\right): \delta 47.9$ $\left(\mathrm{d}, J_{13 C-13 C}=32.6 \mathrm{~Hz}\right), 22.6\left(\mathrm{~d}, J_{13 C-13 C}=32.6 \mathrm{~Hz}\right) ; \operatorname{HRMS}-F A B(m / z):[\mathrm{M}+\mathrm{H}]^{+}$calc'd for $\left[\mathrm{C}_{5} \mathrm{H}_{12} \mathrm{NO}^{13} \mathrm{C}_{2}\right]^{+}, 128.0986$; observed 128.0960.

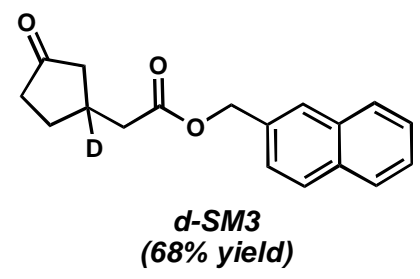

Naphthalen-2-ylmethyl 2-(3-oxocyclopentyl)-d-ethanoate (d-SM3). Prepared as above to afford $\boldsymbol{d}$-SM3 (0.3283 g, $1.15 \mathrm{mmol}, 68 \%$ yield) as a light yellow oil. $\left.{ }^{1} \mathrm{H} \mathrm{NMR} \mathrm{(300} \mathrm{MHz,} \mathrm{CDCl}_{3}\right)$ : $\delta$ 7.87-7.82 (comp. m, 4H), 7.53-7.49 (comp. m, 2H), 7.45 (dd, J = 8.5, $1.6 \mathrm{~Hz}, 1 \mathrm{H}), 5.30$ (s, 2H), $2.53(\mathrm{dd}, J=16.6,16.6 \mathrm{~Hz}, 2 \mathrm{H}), 2.48$ (d, $J=18.6 \mathrm{~Hz}, 1 \mathrm{H}), 2.37-2.11$ (comp. m, 3H), 1.89 (d, $J=$ 
$18.6 \mathrm{~Hz}, 1 \mathrm{H}), 1.64-1.51(\mathrm{~m}, 1 \mathrm{H}) ;{ }^{13} \mathrm{C} \mathrm{NMR}\left(75 \mathrm{MHz}, \mathrm{CDCl}_{3}\right): \delta 218.4,172.0,133.3(2 \mathrm{C}), 128.6$, 128.1, 127.9, 127.6, 126.5 (2C), 126.0, 66.7, 44.6, 39.7, 38.4, $33.2\left(\mathrm{t}, J_{C D}=20.2 \mathrm{~Hz}\right), 29.2 ;{ }^{2} \mathrm{H}$ NMR (76 MHz, $\left.\mathrm{CHCl}_{3}\right): \delta 2.66(\mathrm{~s})$; HRMS-EI $(\mathrm{m} / \mathrm{z}):[\mathrm{M}]^{+\bullet}$ calc'd for $\left[\mathrm{C}_{18} \mathrm{H}_{17} \mathrm{O}_{3}{ }^{2} \mathrm{H}\right]^{+\bullet}, 283.1319$; found 283.1323. All other characterization data are identical to above.

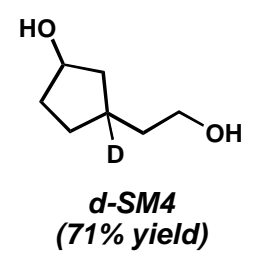

3- $\boldsymbol{d}$-(2-hydroxyethyl)cyclopentanol ( $\boldsymbol{d}$-SM4). Prepared as above to afford $\sim 1: 1$ mixture of diastereomers of $\boldsymbol{d}$-SM4 (46.0 mg, $0.351 \mathrm{mmol}, 71 \%$ yield) as a colorless oil. ${ }^{1} \mathrm{H}$ NMR (300 MHz, $\left.\mathrm{CD}_{3} \mathrm{OD}\right): \delta 4.26(\mathrm{ddd}, J=8.2,3.5,2.4 \mathrm{~Hz}, 0.45 \mathrm{H}), 4.21(\mathrm{ddd}, J=11.3,6.4,6.4 \mathrm{~Hz}, 0.55 \mathrm{H}), 3.56$ $(\operatorname{app~t}, J=6.9 \mathrm{~Hz}, 2 \mathrm{H}), 2.13(\mathrm{dd}, J=13.3,6.4 \mathrm{~Hz}, 0.45 \mathrm{H}), 1.99-1.88$ (m, 0.55H), 1.82-1.70 (comp. $\mathrm{m}, 2 \mathrm{H}), 1.59(\mathrm{dt}, J=22.3,6.9,3 \mathrm{H}), 1.44-1.30(\mathrm{~m}, 1 \mathrm{H}), 1.14(\mathrm{dd}, J=12.0,4.5 \mathrm{~Hz}, 1 \mathrm{H}):{ }^{13} \mathrm{C}$ NMR (75 MHz, $\left.\mathrm{CD}_{3} \mathrm{OD}\right): \delta 74.1,62.2,62.1,43.0,42.7,40.5,40.0,35.8,35.6,35.6\left(\mathrm{t}, J_{C D}=19.5 \mathrm{~Hz}\right)$, $34.8\left(\mathrm{t}, J_{C D}=19.5 \mathrm{~Hz}\right), 31.4,31.1 ;{ }^{2} \mathrm{H} \mathrm{NMR}\left(76 \mathrm{MHz}, \mathrm{CH}_{3} \mathrm{OH}\right): \delta 2.14(\mathrm{~s}), 1.87$ (s). All other characterization data are identical to above.

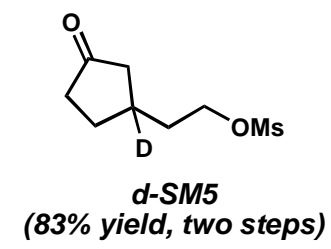

2- $d$-(3-oxocyclopentyl)-ethyl methanesulfonate (d-SM5). Prepared as above to afford $\boldsymbol{d}$ SM5 (60.3 mg, $0.291 \mathrm{mmol}, 83 \%$ yield over two steps) as a colorless oil. ${ }^{1} \mathrm{H}$ NMR (300 MHz, $\left.\mathrm{CDCl}_{3}\right): \delta 4.30$ (app dt, $\left.J=6.1,2.7 \mathrm{~Hz}, 2 \mathrm{H}\right), 3.03(\mathrm{~s}, 3 \mathrm{H}), 2.46$ (d, $\left.J=18.1 \mathrm{~Hz}, 1 \mathrm{H}\right), 2.40-2.12$ (comp. m, 3H), 1.91 (app t, $J=6.4 \mathrm{~Hz}, 2 \mathrm{H}), 1.85$ (d, $J=18.6 \mathrm{~Hz}, 1 \mathrm{H}), 1.61-1.50(\mathrm{~m}, 1 \mathrm{H}) ;{ }^{13} \mathrm{C}$ NMR $\left(75 \mathrm{MHz}, \mathrm{CDCl}_{3}\right): \delta 218.3,68.1,44.7,38.5,37.7,34.9,33.4\left(\mathrm{t}, J_{C D}=19.8 \mathrm{~Hz}\right), 29.3 ;{ }^{2} \mathrm{H} \mathrm{NMR}(76$ $\left.\mathrm{MHz}, \mathrm{CHCl}_{3}\right): \delta 2.35$ (s); HRMS-EI $(\mathrm{m} / \mathrm{z}):[\mathrm{M}]^{+\bullet}$ calc'd for $\left[\mathrm{C}_{8} \mathrm{H}_{13} \mathrm{O}_{4} \mathrm{~S}^{2} \mathrm{H}\right]^{+\bullet}, 207.0676$; found 207.0673. All other characterization data are identical to above. 


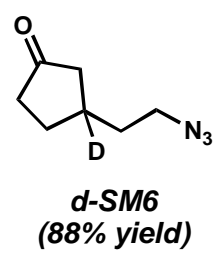

3- $\boldsymbol{d}$-(2-azidoethyl)cyclopentanone (d-SM6). Prepared as above to afford $\boldsymbol{d}$-SM6 (39.3 mg, $0.255 \mathrm{mmol}, 88 \%$ yield) as a colorless oil. ${ }^{1} \mathrm{H} \mathrm{NMR}\left(300 \mathrm{MHz}, \mathrm{CDCl}_{3}\right): \delta 3.36(\mathrm{ddd}, J=6.8,6.8$, $2.9 \mathrm{~Hz}, 2 \mathrm{H}), 2.43(\mathrm{~d}, J=18.3 \mathrm{~Hz}, 1 \mathrm{H}), 2.36-2.30(\mathrm{~m}, 1 \mathrm{H}), 2.23-2.14$ (comp. m, 2H), $1.83(\mathrm{~d}, J=$ $18.1 \mathrm{~Hz}, 1 \mathrm{H}), 1.755(\mathrm{ddd}, J=6.8,6.8,3.7 \mathrm{~Hz}, 2 \mathrm{H}), 1.58-1.51(\mathrm{~m}, 1 \mathrm{H}) ;{ }^{13} \mathrm{C} \mathrm{NMR}(125 \mathrm{MHz}$, $\left.\mathrm{CDCl}_{3}\right): \delta 218.6,50.0,44.8,38.5,34.6,34.3\left(\mathrm{t}, J_{C D}=19.8 \mathrm{~Hz}\right), 29.4 ;{ }^{2} \mathrm{H} \mathrm{NMR}\left(76 \mathrm{MHz}, \mathrm{CHCl}_{3}\right): \delta$ 2.29 (s);. All other characterization data are identical to above.

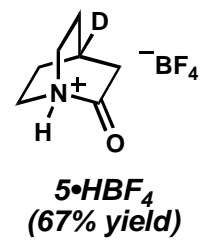

4- $\boldsymbol{d}$-2-quinuclidonium tetrafluoroborate $\left(\mathbf{5} \cdot \mathbf{H B F}_{4}\right)$. Prepared as above to afford $\mathbf{5} \cdot \mathbf{H B F}$ (33.0 mg, $0.154 \mathrm{mmol}, 67 \%$ yield) as white needles. ${ }^{1} \mathrm{H} \mathrm{NMR}\left(300 \mathrm{MHz}, \mathrm{CD}_{3} \mathrm{CN}\right): \delta 7.95$ (br s, 1H), 3.78-3.58 (m, 4H), $2.96(\mathrm{~s}, 2 \mathrm{H}), 2.00-1.95(\mathrm{~m}, 4 \mathrm{H}) ;{ }^{2} \mathrm{H}$ NMR (76 MHz, $\left.\mathrm{CH}_{3} \mathrm{CN}\right): \delta 2.49(\mathrm{~s})$; HRMS-FAB $(m / z):[\mathrm{M}+\mathrm{H}]^{+}$calc'd for $\left[\mathrm{C}_{7} \mathrm{H}_{11} \mathrm{NO}^{2} \mathrm{H}\right]^{+}, 127.0982$; observed 127.0943.

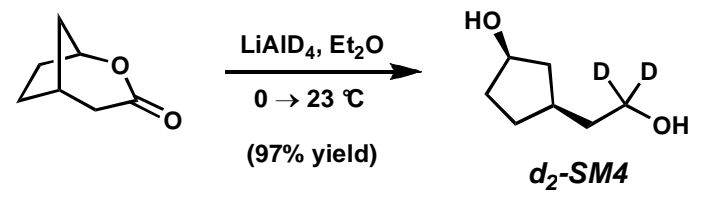

3-(2- $\boldsymbol{d}_{\mathbf{2}}$-hydroxyethyl)cyclopentanol $\left(\boldsymbol{d}_{\mathbf{2}}\right.$-SM4). ${ }^{\mathrm{vi}} \quad$ Prepared by a known method using LiAlD $_{4}$. Diol $\boldsymbol{d}_{\mathbf{2}}$-SM4 isolated as a colorless oil $(0.6317 \mathrm{~g}, 4.78 \mathrm{mmol}, 97 \%$ yield $)$ with $>98 \% d$ incorporation. ${ }^{1} \mathrm{H}$ NMR $\left(300 \mathrm{MHz}, \mathrm{CDCl}_{3}\right): \delta 4.21$ (dddd, $\left.J=5.8,5.8,5.8,5.8 \mathrm{~Hz}, 1 \mathrm{H}\right), 2.14$ (ddd, $J=13.6,7.2,7.2 \mathrm{~Hz}, 1 \mathrm{H}), 2.01-1.85$ (m, 1H), 1.83-1.70 (comp. m, 2H), 1.64-1.55 (comp. m, 3H), $1.48-1.31(\mathrm{~m}, 1 \mathrm{H}), 1.15(\mathrm{dddd}, J=14.4,9.6,5.6,0.5 \mathrm{~Hz}, 1 \mathrm{H}) ;{ }^{13} \mathrm{C} \mathrm{NMR}\left(75 \mathrm{MHz}, \mathrm{CDCl}_{3}\right): \delta 74.1$, 61.4, 42.9, 40.4, 36.0, 35.8, 31.2; ${ }^{2} \mathrm{H}$ NMR (76 MHz, $\left.\mathrm{CH}_{3} \mathrm{OH}\right): \delta 3.51$ (s); HRMS-EI (m/z): [M] ${ }^{+\bullet}$ 
calc'd for $\left[\mathrm{C}_{7} \mathrm{H}_{12} \mathrm{O}_{2}{ }^{2} \mathrm{H}_{2}\right]^{+\bullet}, 132.1119$; found 132.1113. All other characterization data are identical to above.

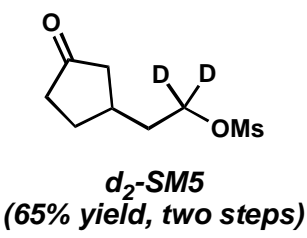

2-(3-oxocyclopentyl)- $\boldsymbol{d}_{2}$-ethyl methanesulfonate $\left(\boldsymbol{d}_{\mathbf{2}}\right.$-SM5). Prepared as above to afford $\boldsymbol{d}_{2}$-SM5 (0.3775 g, $1.81 \mathrm{mmol}, 65 \%$ yield over two steps) as a colorless oil. ${ }^{1} \mathrm{H}$ NMR (300 MHz, $\left.\mathrm{CDCl}_{3}\right): \delta 3.01(\mathrm{~s}, 3 \mathrm{H}), 2.49-2.40(\mathrm{~m}, 1 \mathrm{H}), 2.39-2.10$ (comp. m, 4H), 1.89 (d, J = 6.9 Hz, 2H), 1.83 $(\mathrm{ddd}, J=17.5,7.4,1.3 \mathrm{~Hz}, 1 \mathrm{H}), 1.63-1.47(\mathrm{~m}, 1 \mathrm{H}) ;{ }^{13} \mathrm{C} \mathrm{NMR}\left(75 \mathrm{MHz}, \mathrm{CDCl}_{3}\right): \delta 218.3,67.6$ (pentet, $\left.J_{C D}=22.7 \mathrm{~Hz}\right), 44.8,38.4,37.6,34.7,33.7,29.4 ;{ }^{2} \mathrm{H} \mathrm{NMR}\left(76 \mathrm{MHz}, \mathrm{CHCl}_{3}\right): \delta 4.29(\mathrm{~s})$; HRMS-EI $(\mathrm{m} / \mathrm{z})$ : $[\mathrm{M}]^{+\bullet}$ calc'd for $\left[\mathrm{C}_{8} \mathrm{H}_{12} \mathrm{SO}_{4}{ }^{2} \mathrm{H}_{2}\right]^{+\bullet}$, 208.0738; found 208.0741. All other characterization data are identical to above.

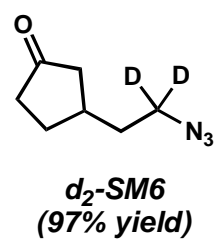

3-(2- $\boldsymbol{d}_{\mathbf{2}}$-azidoethyl)cyclopentanone $\left(\boldsymbol{d}_{\mathbf{2}}\right.$-SM6). Prepared as above to afford $\boldsymbol{d}_{\mathbf{2}}$-SM6 (143.6 mg, 0.925 mmol, 97\% yield) as a colorless oil. ${ }^{1} \mathrm{H}$ NMR (300 $\left.\mathrm{MHz}, \mathrm{CDCl}_{3}\right): \delta 2.48-2.39$ (m, 1H), 2.37-2.11 (comp. m, 4H), 1.83 (ddd, $J=17.6,9.8,1.3 \mathrm{~Hz}, 1 \mathrm{H}), 1.74$ (d, $J=6.7 \mathrm{~Hz}, 2 \mathrm{H}), 1.61-1.50$ $(\mathrm{m}, 1 \mathrm{H}) ;{ }^{13} \mathrm{C}$ NMR $\left(75 \mathrm{MHz}, \mathrm{CDCl}_{3}\right.$ ): $\delta 218.6,49.3$ (pentet, $J_{C D}=21.7 \mathrm{~Hz}$ ), 44.8, 38.5, 34.6, 34.4, 29.4; ${ }^{2} \mathrm{H} \mathrm{NMR}\left(76 \mathrm{MHz}, \mathrm{CHCl}_{3}\right): \delta 3.32(\mathrm{~s})$. All other characterization data are identical to above.

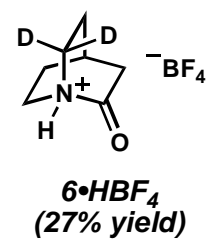


6,6- $\boldsymbol{d}_{2}$-2-quinuclidonium tetrafluoroborate $(\mathbf{6} \cdot \mathbf{H B F})_{4}$. Prepared as above to afford $\mathbf{X X} \cdot \mathbf{B F}_{4}\left(15.3 \mathrm{mg}, 0.0712 \mathrm{mmol}, 27 \%\right.$ yield) as white needles. ${ }^{1} \mathrm{H}$ NMR $\left(300 \mathrm{MHz}, \mathrm{CD}_{3} \mathrm{CN}\right): \delta$ 7.96 (br s, 1H), 3.77-3.58 (m, 2H), 2.97 (d, $J=3.2 \mathrm{~Hz}, 2 \mathrm{H}), 2.51$ (app. pentet, $J=3.2 \mathrm{~Hz}, 1 \mathrm{H}$ ), 2.15 $(\mathrm{m}, 2 \mathrm{H}), 2.02-1.94(\mathrm{~m}, 2 \mathrm{H}) ;{ }^{2} \mathrm{H}$ NMR $\left(76 \mathrm{MHz}, \mathrm{CH}_{3} \mathrm{CN}\right): \delta 3.68(\mathrm{~s}), 3.59(\mathrm{~s})$; HRMS-FAB $(\mathrm{m} / z)$ : $[\mathrm{M}+\mathrm{H}]^{+}$calc'd for $\left[\mathrm{C}_{7} \mathrm{H}_{10} \mathrm{NO}^{2} \mathrm{H}_{2}\right]^{+}, 128.1044$; observed 128.1042.

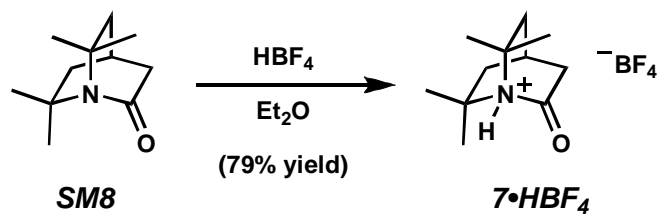

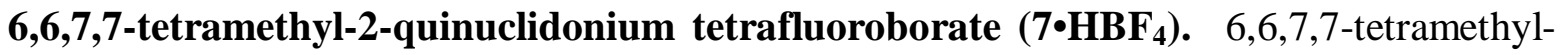
2-quinuclidone (SM8, $24.9 \mathrm{mg}, 0.137 \mathrm{mmol}, 1.0$ equiv) was dissolved in $\mathrm{Et}_{2} \mathrm{O}(1.0 \mathrm{~mL}, 0.14 \mathrm{M})$ and $\mathrm{HBF}_{4}$ in $\mathrm{Et}_{2} \mathrm{O}$ (54 wt \% solution, $38 \mu \mathrm{L}, 0.274 \mathrm{mmol}, 2.0$ equiv) was added in one portion. The reaction was stirred for $30 \mathrm{~min}$ and the precipitate was collected by filtration and dried under vacuum to afford $\mathbf{7} \cdot \mathrm{HBF}_{\mathbf{4}}(31.6 \mathrm{mg}, 0.117 \mathrm{mmol}, 86 \%$ yield) as a tan solid. HRMS-FAB $(\mathrm{m} / \mathrm{z})$ : $[\mathrm{M}+\mathrm{H}]^{+}$calc'd for $\left[\mathrm{C}_{11} \mathrm{H}_{20} \mathrm{NO}\right]^{+}, 182.1545$; observed 182.1552 . 


\section{Computational Results}

Table 1. Summary of Calculated Energies and Corrections

\begin{tabular}{|c|c|c|c|}
\hline \multirow{2}{*}{ Molecule } & Electronic Energy & Scaled zpe/thermal corr. & BSSE \\
\cline { 2 - 4 } & -403.438205 & 0.179704 & \\
\hline \hline $\boldsymbol{1}$ & -403.813593 & 0.194329 & 0.001093 \\
\hline $\begin{array}{c}\boldsymbol{1}+\mathrm{H}^{+} \\
(\mathrm{N}-\text { protonated })\end{array}$ & -403.775347 & 0.191370 & 0.000383 \\
\hline $\boldsymbol{1}+\mathrm{H}^{+}$ & & Hartrees \\
\hline$($ O-protonated $)$ & -560.727058 & 0.294566 & 0.001042 \\
\hline $\boldsymbol{7}$ & -561.116387 & 0.308837 & \\
\hline $\mathbf{7}+\mathrm{H}^{+}$ & & & \\
\hline
\end{tabular}

Table 2. Summary of Calculated Proton Affinities including ZPE, Thermal, and BSSE Corrections

\begin{tabular}{|c|c|c|c|}
\hline & $\boldsymbol{l}$ (N-protonation) & $\boldsymbol{1}$ (O-protonation) & $\boldsymbol{7}$ (N-protonation) \\
\hline \hline PA $(\mathrm{kJ} / \mathrm{mol})$ & 944.3 & 853.5 & 982.0 \\
\hline
\end{tabular}

Optimized structures. Bond lengths below are given in angstroms.

Optimized Structure of 2-quinuclidone

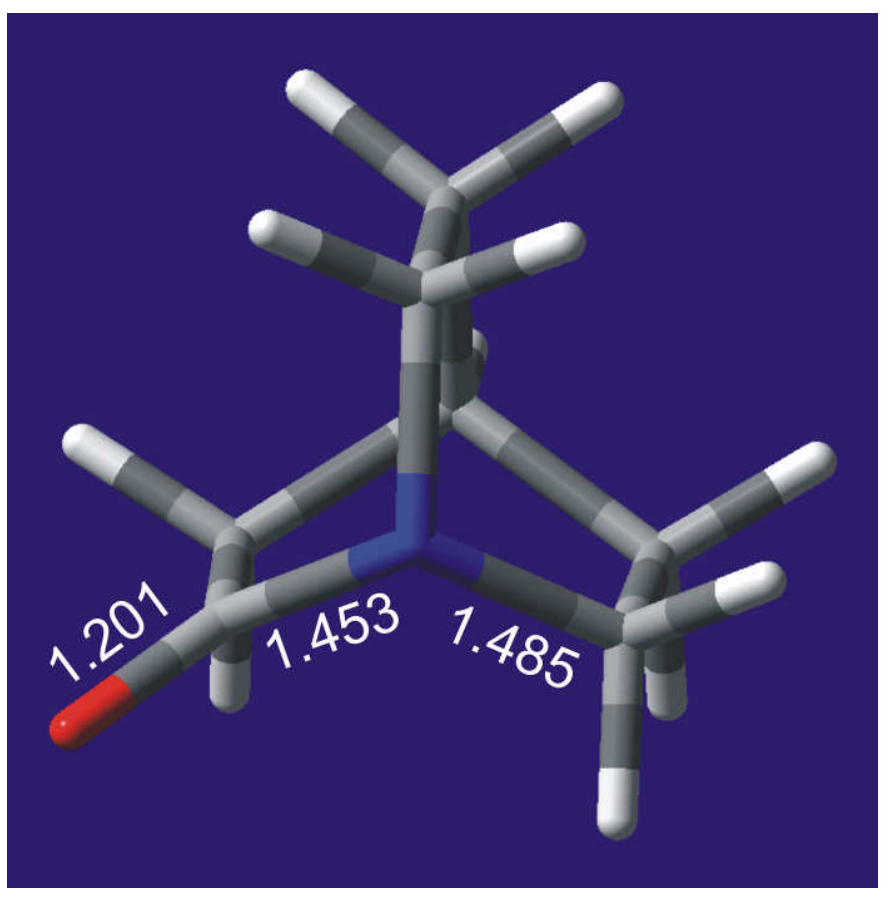

$\mathrm{O}=\mathrm{C}-\mathrm{N}-\mathrm{C}$ dihedral angles: $\pm 121^{\circ}$ 
Optimized Structure of protonated 2-quinuclidone.

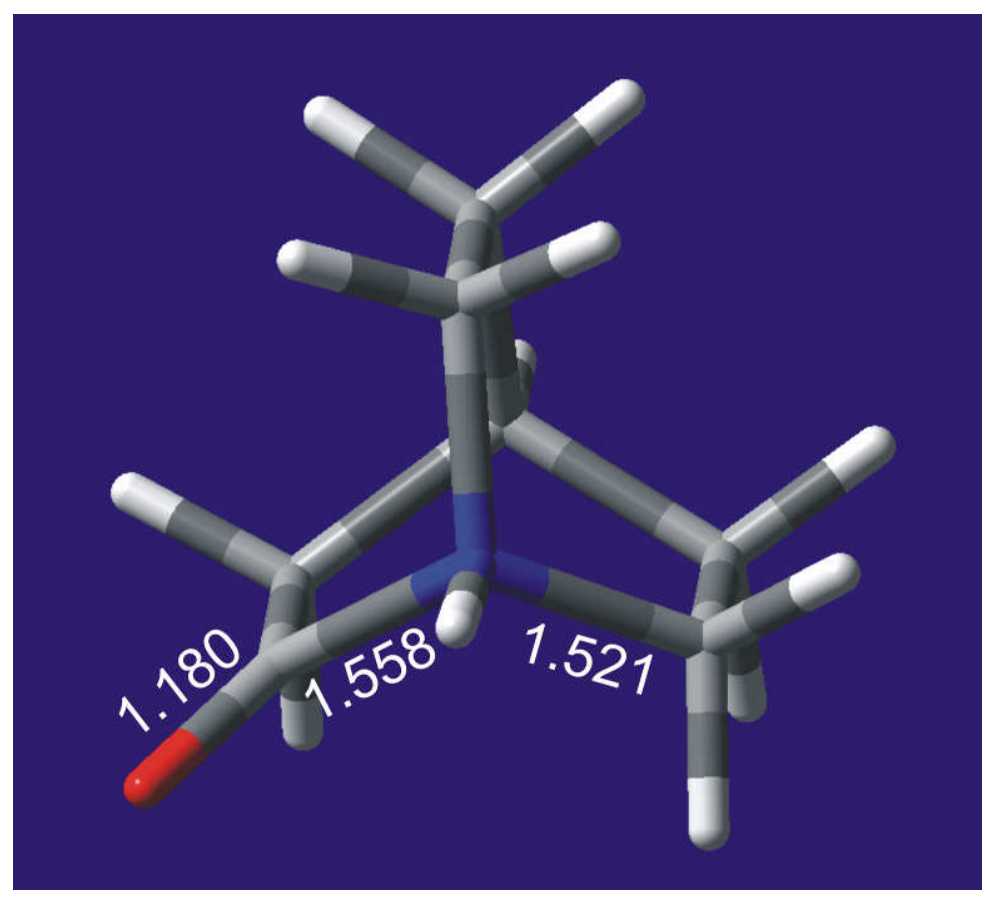

$\mathrm{O}=\mathrm{C}-\mathrm{N}-\mathrm{C}$ dihedral angles $\pm 119^{\circ},-0.0^{\circ}$

Optimized Structure of Tetramethyl 2-quinuclidone.

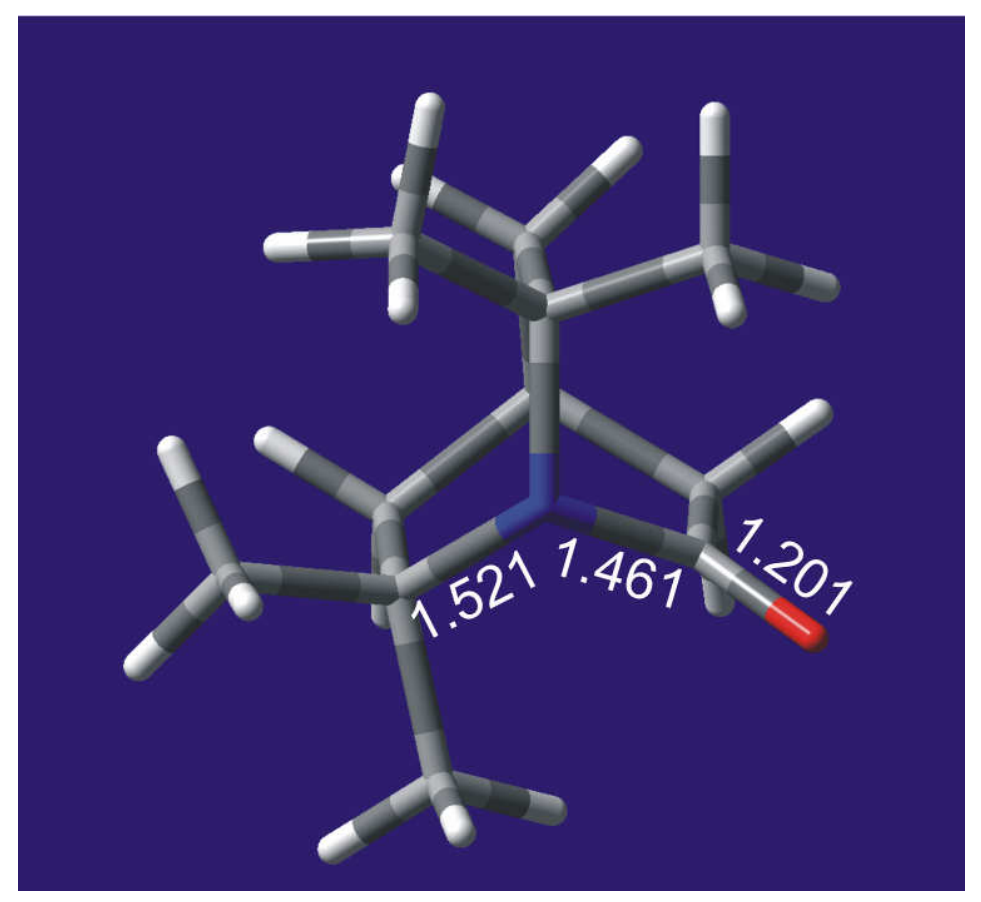

$\mathrm{O}=\mathrm{C}-\mathrm{N}-\mathrm{C}$ dihedral angles: $\pm 118^{\circ}$ 
Optimized Structure of Protonated Tetramethyl 2-quinuclidone.

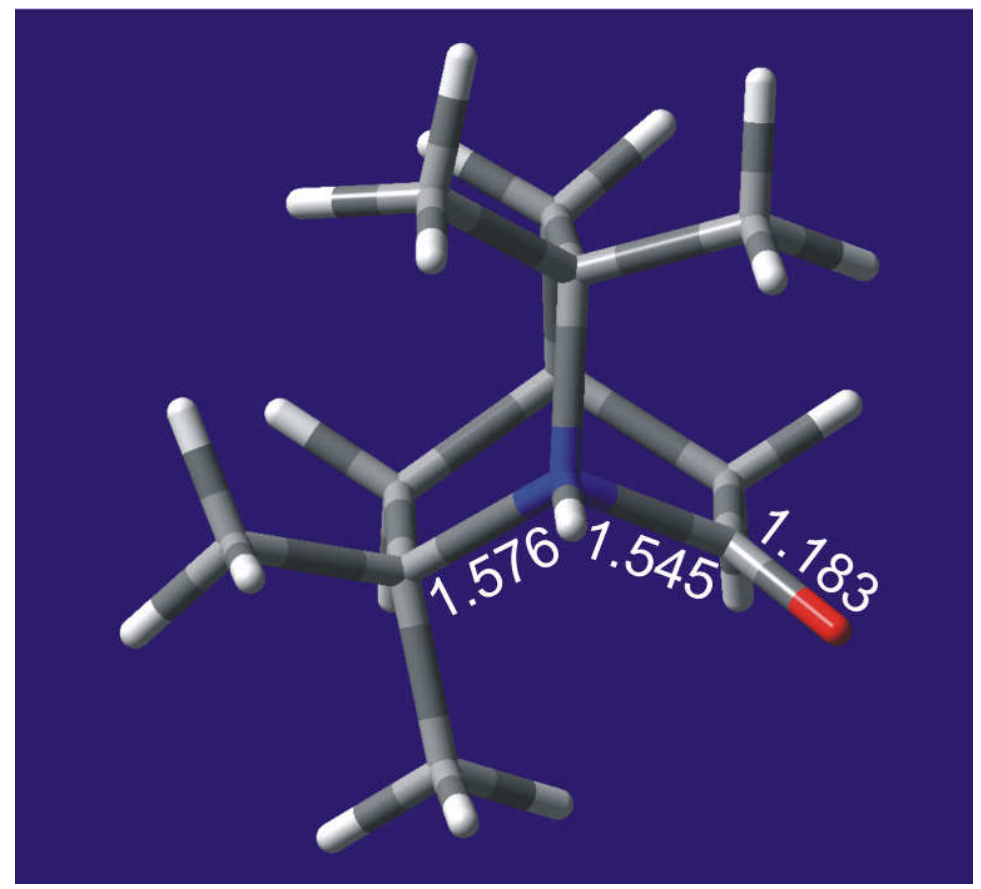

$\mathrm{O}=\mathrm{C}-\mathrm{N}-\mathrm{C}$ dihedral angles: $\pm 116^{\circ}, 0.0^{\circ}$ 
$\mathrm{MS}^{2}$ spectra of 3, 4, 5, 6 and their hydrolysis products. Fragmentation patterns of isotopically labeled compounds are in agreement with mechanism proposed in Scheme 1.
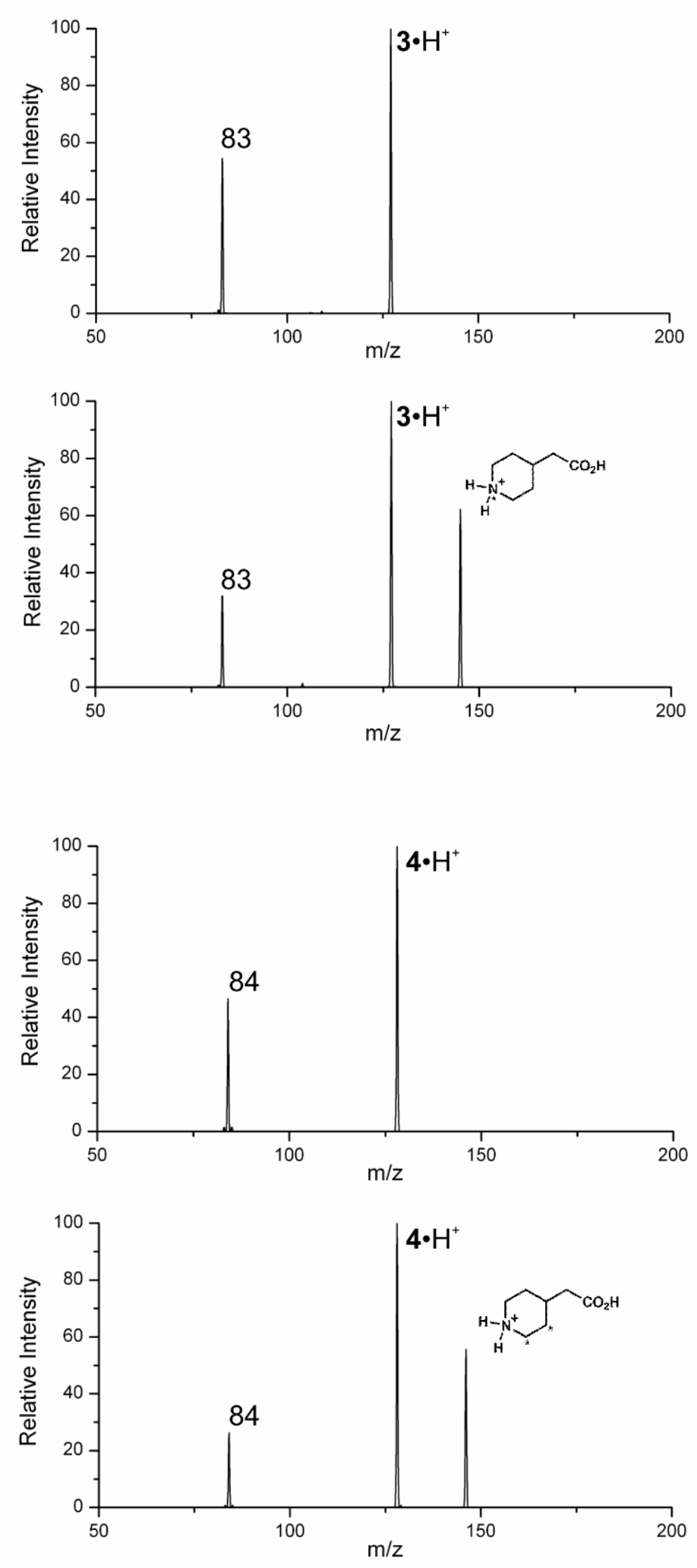

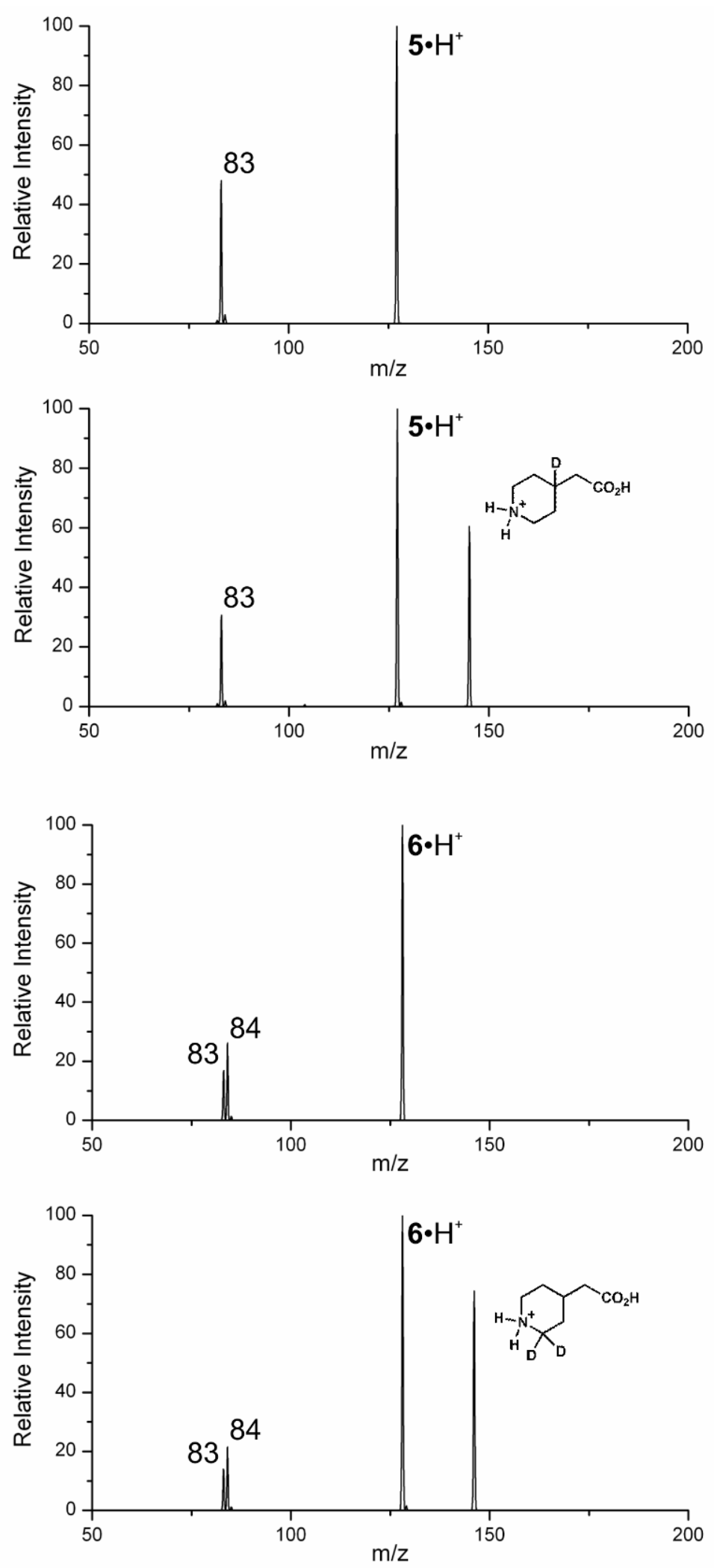


\section{Extended Kinetic Method Plots}

Second extended kinetic plot with direct entropy correction. The best-fit y-intercepts from the first entropy corrected kinetic plot shown in Figure 1 were plotted against the corresponding slopes at $18 \%, 25 \%, 35 \%, 50 \%$, and $85 \%$ normalized collision energies. The slope of the line shown below is equal to $\left[\mathrm{PA}_{\text {quin }}-\mathrm{PA}_{\mathrm{avg}}\right]$.

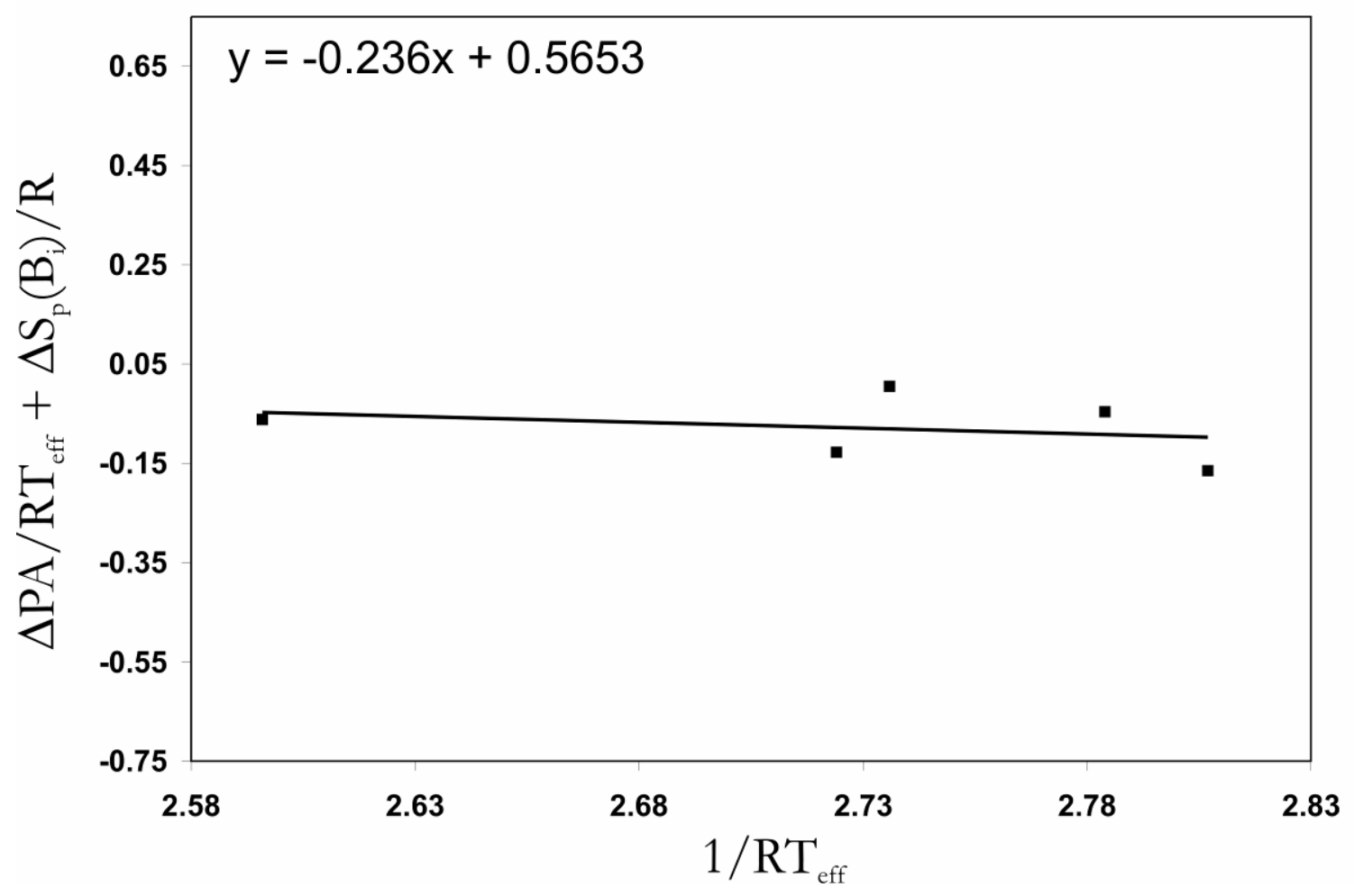


Entropy corrected kinetic plot of bulky bases. The second trend observed is associated with the proton affinity of the carbonyl oxygen of 2-quinuclidone. Bulky bases a-e: 3-aminopyridine; 3,5lutidine; diisobutylamine; 2,4-lutidine; 1,4-diazabicyclo[2.2.2]-octane.

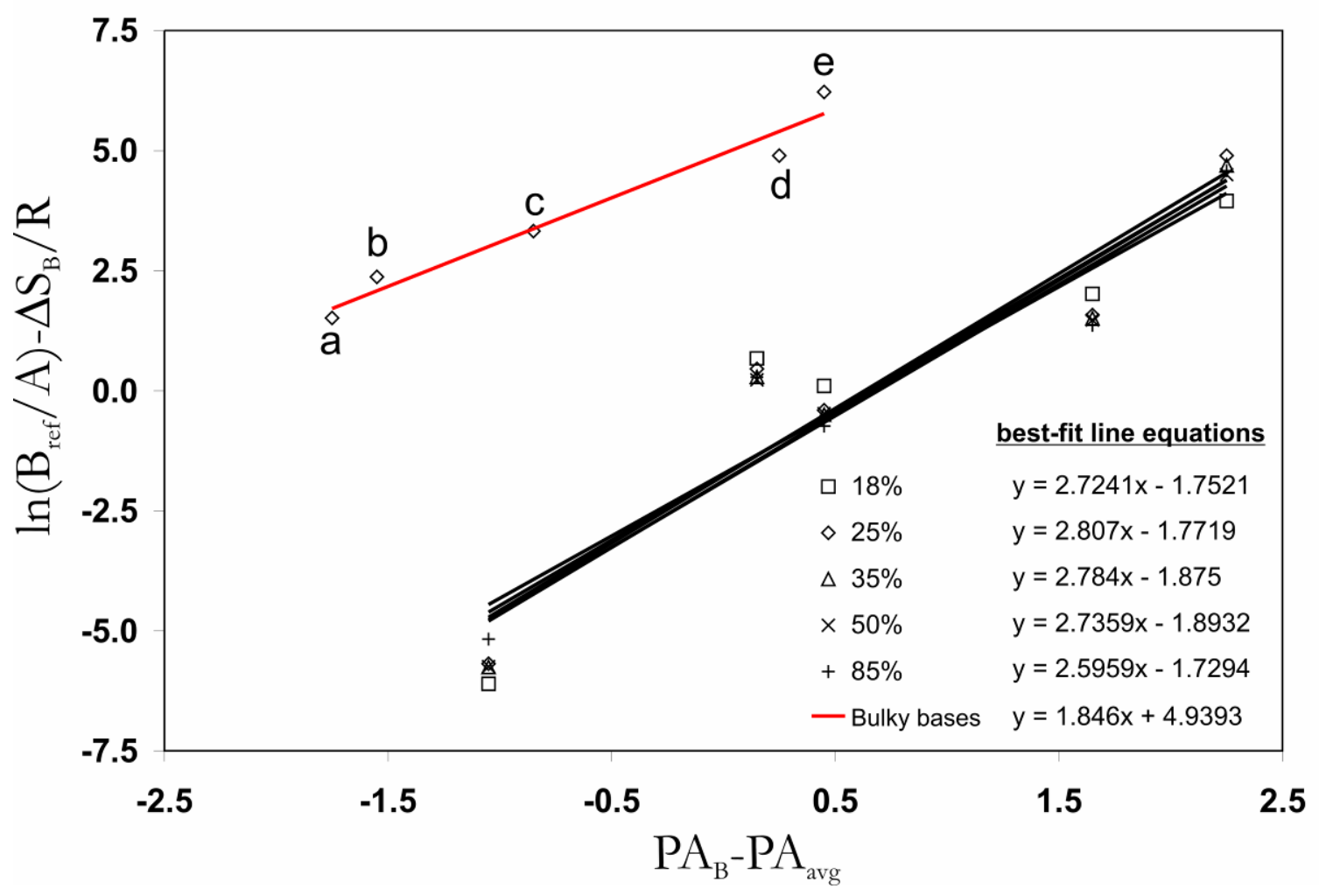

i (a) Gannon, W. F.; House, H. O. Org. Synth. 1973, CV 5, 294-196. (b) Dauphin, G.; Gramain, J.C.; Kergomard, A.; Renard, M. F.; Veschambre, H. J. Chem. Soc., Chem. Commun. 1980, 318-319. ii Greenburg, A.; Wu, G.; Tsai, J.-C.; Chiu, Y.-Y. Struct. Chem. 1993, 4, 127-129.

iii Andersson, M. P.; Uvdal, P. J. Phys. Chem. A 2005, 109, 2937-2941.

${ }^{\text {iv }}$ Boys, S. F.; Bernardi, F. Mol. Phys. 1970, 19, 553-566.

v (a) Tomioka, H.; Oshima, K.; Nozaki, H. Tetrahedron Lett. 1982, 23, 539-542. (b) Kanemoto, S.; Tomioka, H.; Oshima, K.; Nozaki, H. Bull. Chem. Soc. Jpn. 1986, 59, 105-108.

${ }^{\mathrm{vi}}$ Tani, K.; Stoltz, B. M. Nature 2006, 441, 731-734 (supporting information). 